\title{
Combined transcriptome and metabolome analyses to understand the dynamic responses of rice plants to attack by the rice stem borer Chilo suppressalis (Lepidoptera: Crambidae)
}

\author{
Qingsong Liu' ${ }^{1+}$, Xingyun Wang ${ }^{1 \dagger}$, Vered Tzin², Jörg Romeis ${ }^{1,3}$, Yufa Peng ${ }^{1}$ and Yunhe $\mathrm{Li}^{1^{*}}$
}

\begin{abstract}
Background: Rice (Oryza sativa L.), which is a staple food for more than half of the world's population, is frequently attacked by herbivorous insects, including the rice stem borer, Chilo suppressalis. C. suppressalis substantially reduces rice yields in temperate regions of Asia, but little is known about how rice plants defend themselves against this herbivore at molecular and biochemical level.

Results: In the current study, we combined next-generation RNA sequencing and metabolomics techniques to investigate the changes in gene expression and in metabolic processes in rice plants that had been continuously fed by C. suppressalis larvae for different durations $(0,24,48,72$, and 96 h). Furthermore, the data were validated using quantitative real-time PCR. There were 4,729 genes and 151 metabolites differently regulated when rice plants were damaged by C. suppressalis larvae. Further analyses showed that defense-related phytohormones, transcript factors, shikimate-mediated and terpenoid-related secondary metabolism were activated, whereas the growth-related counterparts were suppressed by C. suppressalis feeding. The activated defense was fueled by catabolism of energy storage compounds such as monosaccharides, which meanwhile resulted in the increased levels of metabolites that were involved in rice plant defense response. Comparable analyses showed a correspondence between transcript patterns and metabolite profiles.

Conclusion: The current findings greatly enhance our understanding of the mechanisms of induced defense response in rice plants against C. suppressalis infestation at molecular and biochemical levels, and will provide clues for development of insect-resistant rice varieties.
\end{abstract}

Keywords: Oryza sativa, Induced response, Next generation sequencing, Plant-insect interactions, Phytohormones, Phenylpropanoids, Carbohydrates, Amino acids, Terpenoids

\section{Background}

To protect against attack by herbivorous insects, plants have evolved both constitutive and induced defense mechanisms [1]. Induced defenses include both direct and indirect responses, which are activated by herbivore

\footnotetext{
* Correspondence: liyunhe@caas.cn

${ }^{\dagger}$ Equal contributors

'State Key Laboratory for Biology of Plant Diseases and Insect Pests, Institute of Plant Protection, Chinese Academy of Agricultural Sciences, Beijing, China Full list of author information is available at the end of the article
}

feeding, crawling, frass, or oviposition [2]. Induced direct responses involve the production of secondary metabolites and insecticidal proteins, which can reduce herbivore development and survival $[1,3]$. While induced indirect responses mainly involve the release of volatile chemicals that can attract natural enemies of herbivores $[1,3,4]$.

Plant response against herbivory are associated with large-scale changes in gene expression and metabolism [5-9]. The integration of modern omics technologies such as transcriptomics, proteomics, and metabolics

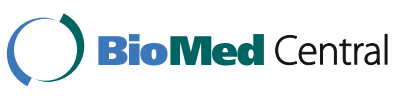


provides a great opportunity for a deeper understanding of the mechanisms of plant defence responses to herbivore feeding at molecular and cellular levels [7, 9-11]. Previous results have suggested that plant response to herbivore feeding is a dynamic process, and that the transcript patterns, protein and metabolite profiles are temporally and spatially regulated $[1,10,12]$. This suggests that it is essential to investigate the dynamic at transcriptional, proteomic and metabolic changes associated to insect feeding $[6,7,9,11]$. Transcriptomic and proteomic studies are only able to predict changes in gene expression and the protein level, while metabolomic studies investigate the changed functions exerted by these genes or proteins. Therefore, the integration of transcriptomic, proteomic, and metabolic approaches can gain a better understanding of plant responses to herbivore feeding [10].

Rice (Oryza sativa L.) is the staple food for more than half of the world's population [13], but rice yield is frequently reduced by herbivorous insects [14]. Lepidopteran stem borers are chronic pests in all rice ecosystems, and the rice stem borer Chilo suppressalis is among the most serious rice pest in temperate regions of Asia [15]. C. suppressalis is particularly damaging in China because of the wide adoption of hybrid varieties. A better understanding of the genetic and molecular mechanisms underlying rice plant defense against insect pests is important for developing resistant rice varieties and other strategies for controlling pests [14]. The genetic basis of rice defense against piercingsucking planthoppers has been well elucidated, and several gene functions have been identified [16-19]. For example, Liu et al. [16] identified several lectin receptor kinase genes that confer durable resistance to the brown planthopper Nilaparvata lugens and the white back planthopper Sogatella furcifera. However, the defense response of rice plants to chewing insects, such as lepidopteran larvae, has rarely been studied, although a few studies have been conducted using microarray technology, in which a relatively small number of differentially expressed genes were identified $[8,20,21]$. In addition, the previous experiments were conducted with rice samples collected at only one time point after C. suppressalis infestation, and the data did not therefore reveal the dynamic response of rice plants to $C$. suppressalis feeding at transcriptional and metabolic levels.

In the current study, we combined transcriptome and metabolome analyses to investigate the dynamic responses of rice plants to attack by C. suppressalis, with the expectation to provide a better understanding of rice defense mechanisms to $C$. suppressalis infestation and clues for the development of rice pest control strategies.

\section{Methods}

\section{Plants and growing conditions}

The rice cultivar Minghui 63, an elite indica restorer line for cytoplasmic male sterility in China, was used in this study. Seeds were incubated in water for 2 day and sown in a seedling bed in a greenhouse $\left(27 \pm 3{ }^{\circ} \mathrm{C}, 65 \pm 10 \% \mathrm{RH}\right.$, 16 L: 8 D). Fifteen-day-old seedlings were individually transplanted into plastic pots $\left(630 \mathrm{~cm}^{3}\right)$ containing a mixture of peat and vermiculite (3:1). Plants were watered daily and supplied with $10 \mathrm{ml}$ of nitrogenous fertilizer every week. Plants were used for the experiments four weeks after transplanting.

\section{Insect colony}

Specimens of $C$. suppressalis were retrieved from a laboratory colony that had been maintained on an artificial diet for over 60 generations with annual introductions of field-collected individuals. The colony was maintained at $27 \pm 1{ }^{\circ} \mathrm{C}$ with $75 \pm 5 \% \mathrm{RH}$ and a $16 \mathrm{~L}: 8 \mathrm{D}$ photoperiod [22].

\section{Insect bioassay}

Potted rice plants were transferred to a climate control chamber $\left(27 \pm 1{ }^{\circ} \mathrm{C}, 75 \pm 5 \% \mathrm{RH}, 16 \mathrm{~L}: 8 \mathrm{D}\right.$ photoperiod $)$ for $24 \mathrm{~h}$ and were then infested with three $3^{\text {rd }}$-instar $C$. suppressalis per plant. The larvae had been starved for $2 \mathrm{~h}$ before they were caged with the rice plants. The main rice stems, $4 \mathrm{~cm}$ above the area damaged by the larvae, were harvested after they had been exposed to $C$. suppressalis feeding for 0 (healthy, control rice plants), 24, 48, 72, and $96 \mathrm{~h}$. Plant samples were immediately frozen in liquid nitrogen and stored at $-80{ }^{\circ} \mathrm{C}$ for later analyses. Four samples (replicates) were collected at each of the following time points and were used for transcriptome analysis: 0 , 24,48 , and $72 \mathrm{~h}$. Ten samples were collected at each of the following time points and were used for metabolome analyses: $0,48,72$, and $96 \mathrm{~h}$. The sampling time points differed for the transcriptome and metabolome analyses because the rice plants were expected to respond faster to insect feeding on the transcriptomic level than on the metabolomic level $[1,10]$.

\section{Transcriptome analysis RNA extraction}

The total RNA from the rice stem samples was isolated using TRIzol reagent (Invitrogen, Carlsbad, CA, USA) according to the manufacturer's instructions. RNA quality was checked with a 2200 Bioanalyzer (Agilent Technologies, Inc., Santa Clara, CA, USA). The assessment showed that the RNA integrity number (RIN) of all samples was $>9.7$. 


\section{Library preparation and RNA-sequencing}

The sequencing library of each RNA sample was prepared using Ion Total RNA-sequencing (RNA-Seq) Kit v2 (Life Technologies, Carlsbad, CA, USA) according to the manufacturer's protocols. In brief, mRNA was purified from $5 \mu \mathrm{g}$ of total RNA from each sample with oligo (dT) magnetic beads and was fragmented using RNase III (Invitrogen, Carlsbad, CA, USA). The fragmented mRNA was hybridized and ligated with Ion adaptor. The first-strand cDNA strand was synthesized using reverse transcription of random primers, which was followed by second-strand cDNA synthesis using DNA polymerase I and RNase $\mathrm{H}$ (Invitrogen, Carlsbad, CA, USA). The resulting cDNA fragments underwent an end repair process followed by phosphorylation and then ligation of adapters. These products were subsequently purified and amplified by PCR to create cDNA libraries. The cDNA libraries were processed and enriched on a OneTouch 2 instrument using Ion $\mathrm{PI}^{\mathrm{mx}}$ Template OT2 200 Kit (Life Technologies, Carlsbad, CA, USA) to prepare the Template-Positive Ion PI ${ }^{\mathrm{TW}}$ Ion Sphere $^{\mathrm{Tm}}$ Particles. After enrichment, the mixed Template-Positive Ion $\mathrm{PI}^{\mathrm{Tm}}$ Ion Sphere ${ }^{\mathrm{Tm}}$ Particles were finally loaded on the Ion $\mathrm{PI}^{\mathrm{Im}}$ Chip and sequenced using the Ion $\mathrm{PI}^{\mathrm{ix}}$ Sequencing 200 Kit (Life Technologies, Carlsbad, CA, USA). Bioinformatics data analyses of the RNA-seq libraries were performed by Shanghai Novelbio Ltd. as previously described [23].

\section{Quantitative real-time $P C R$}

The plant tissue samples for quantitative real-time PCR (qPCR) were collected from different plants of the same batch of rice plants that were sampled for RNA-seq experiments. In brief, $500 \mathrm{ng}$ of total RNA was reverse transcribed using a first-strand cDNA synthesis kit (Promega, Madison, WI, USA), digested with DNase I (Thermo Fisher Scientific, Waltham, MA, USA), and then diluted 50X. The qPCR reaction was performed using SYBR Premix Ex Taq Ready Mix with POX reference dye (Takara Biotech, Kyoto, Japan) and an ABI 7500 Real-time PCR Detection System instrument (Applied Biosystems Foster City, CA, USA). The thermocycler setting was as follows: $30 \mathrm{~s}$ at $95^{\circ} \mathrm{C}$, followed by 40 cycles of $5 \mathrm{~s}$ at $95{ }^{\circ} \mathrm{C}$ and $34 \mathrm{~s}$ at $60{ }^{\circ} \mathrm{C}$. To confirm the formation of single peaks and to exclude the possibility of primerdimer and non-specific product formation, a melt curve $\left(15 \mathrm{~s}\right.$ at $95^{\circ} \mathrm{C}, 60 \mathrm{~s}$ at $60^{\circ} \mathrm{C}$, and $15 \mathrm{~s}$ at $95^{\circ} \mathrm{C}$ ) was generated by the end of each PCR reaction. Primer pairs were designed using Beacon Designer software (Premier Biosoft, version 7.0) and are listed in Additional file 1: Table S1. The relative fold-changes of gene expression were calculated using the comparative $2^{-\Delta \Delta C T}$ method [24] and were normalized to the housekeeping gene ubiquitin 5 [25]. All
qPCR reactions were repeated in three biological and four technical replications.

\section{Analyses of differentially expressed genes (DEGs)}

RNA-seq read quality values were checked using FASTQC (http://www.bioinformatics.babraham.ac.uk/projects/ fastqc/). The reads were mapped to the reference rice genome of the Michigan State University (MSU) Rice Genome Annotation Project database (RGAP, V7.0) (http://rice.plantbiology.msu.edu/) [26] using MapSplice software [27]. The DEGSeq algorithm [28] was used to filter DEGs. Reads per kilobase of exon model per million mapped reads (RPKM) were used to explore the expression levels of the DEGs [29], and an upper quartile algorithm was applied for data correction. False discovery rate (FDR) was used for the correction of data occur in multiple significant tests [30]. Genes whose expression differed by at least two-fold $\left(\log _{2}\right.$ (fold change) $>1$ or $<-1$, FDR $<0.05)$ were regarded as DEGs as determined with the R statistical programming environment (http://www.rproject.org). The DEGs in rice plants that had been fed by caterpillars for 24,48 , or $72 \mathrm{~h}$ were, respectively, compared to those that had never been fed using MapMan software to get an overview of the metabolism [31]. Venn diagrams were generated using these DEGs to identify common and unique genes affected by C. suppressalis among different time points [32]. Time Series-Cluster analysis, based on the Short Time-series Expression Miner (STEM) method (http://www.cs.cmu.edu/ jernst/stem/) [33], was used to identify the global trends and similar temporal model patterns of the expression of the total DEGs.

\section{Phytohormone signature analyses}

Hormonometer program analyses [34] (http://hormono meter.weizmann.ac.il/) was used to assess the similarity of the expression of rice genes induced by $C$. suppressalis with indexed data sets of those elicited by exogenous application of phytohormones to Arabidopsis as previously described [7]. The rice genes were blasted to the Arabidopsis thaliana genome. The Arabidopsis gene identifies (AGI) were converted to Arabidopsis probe set identifies using the g:Convert Gene ID Converter tool [35] (http://biit.cs.ut.ee/gprofiler/gconvert.cgi). Only genes included in RNA-seq containing Arabidopsis probe set identifies were kept for analyses. In some cases, there were two probe sets for one AGI, while in few cases there were two AGIs for one probe set. This indicates that lines were duplicated and sets were thus discarded.

\section{Gene ontology (GO) and pathway enrichment analyses}

DEGs belonging to different classes were retrieved for GO and pathway analysis. GO analysis was conducted using the GSEABase (gene set enrichment analysis base) package from BioConductor (http://www.bioconductor.org/) 
based on biological process categories (Fisher's exact test, FDR $<0.001)$. Pathway analyses were conducted to elucidate significant pathways of DEGs according to the Kyoto Encyclopedia of Gene and Genomes (KEGG) (http://www.genome.jp/kegg) databases. Fisher's exact test followed by Benjamini-Hochberg multiple testing correction was applied to identify significant pathways $(P<0.05)$.

\section{Metabolome analyses}

Samples were prepared using the automated Microlab STAR system (Hamilton Company, Bonaduz, Switzerland) and were analyzed using ultrahigh performance liquid chromatography-tandem mass spectroscopy (UHPLC-MS) and gas chromatography-mass spectrometry (GC-MS) platforms by Metabolon Inc. (Durham, North Carolina, USA). These platforms have been previously described [36, 37]. In brief, a recovery standard was added before the first step in the extraction process for quality control purposes. Protein fractions of the samples were removed by serial extractions with methanol. The samples were subsequently concentrated on a Zymark TurboVap system (KcKinley Scientific, Sparta, NJ, USA) to remove the organic solvent and then were vacuum dried. The resulting samples were divided into five fractions, and they were used for analyis by: i) UHPLC-MS with positive ion mode electrospray ionization, ii) UHPLC-MS with negative ion mode electrospray ionization, iii) UHPLC-MS polar platform (negative ionization), iv) GC-MS, and v) for being reserved for backup, respectively. Before the UHPLC-MS analysis, the subsamples were stored overnight under nitrogen. For GCMS analysis, each sample was dried under vacuum overnight. UHPLC-MS and GC-MS analyses of all samples were carried out in collaboration with Metabolon Inc. as previous described [36, 37].

For statistical analysis, missing values were assumed to be below the limits of detection, and these values were inputted with a minimum compound value [37]. The relative abundances of each metabolite was log transformed before analysis to meet normality. Dunnett's test was used to compare the abundance of each metabolite between different time points. Statistical analyses were performed using the SPSS 22.0 software package (IBM SPSS, Somers, NY, USA).

\section{Results}

Global transcriptome changes in rice plants during Chilo suppressalis infestation

A total of 16 libraries (four biological replicates of four sampling times) were conducted, resulting in approximately 29-41 million clean reads; GC content accounted for $48-53 \%$ of these reads (Additional file 2: Table S2). The average number of reads that mapped to the rice reference genome was $>87 \%$, and unique mapping rates ranged from 73 to $87 \%$ (Additional file 2: Table S2). The unique matching reads were used for further analysis. Gene structure analysis showed that most of the mapped reads (61-73\%) were distributed in exons (Additional file 3: Table S3). RNA-seq data were normalized to RPKM values to quantify transcript expression. In total, 42,100 genes were detected in all samples (Additional file 4: Table S4). Only significantly changed genes with $P<0.05$ (FDR) and fold-change $>2$ or $<0.05$ were considered to be differentially expressed genes (DEGs), resulting in a total of 4,729 DEGs at a minimum of two time points (Fig. 1, Additional file 5: Table S5 and Additional file 6: Table S6). A comparison of DEGs at the different time points relative to the control ( $24 \mathrm{~h}$ vs. $0 \mathrm{~h}, 48 \mathrm{~h}$ vs. $0 \mathrm{~h}$, and $72 \mathrm{~h}$ vs. $0 \mathrm{~h}$ ) revealed over one thousand genes with significantly altered expression levels, with more genes being up-regulated than down-regulated (Fig. 1a). MapMan analyses showed that the up-regulated DEGs in rice plants between different time-point (24, 48, or $72 \mathrm{~h}$ ) and the control $(0 \mathrm{~h})$ were mainly involved in cell wall, lipid and secondary metabolism. While the down-regulated DEGs mainly involved in light reactions (Additional file 7: Figure S1). A Venn Diagram of this data set indicated that 1,037 genes were differently expressed at all 3 time points of 24,48 , and $72 \mathrm{~h}$ relative to $0 \mathrm{~h}$ (Fig. 1b). However, much lower number of DEGs detected between the time points of $24 \mathrm{~h}$ vs. $48 \mathrm{~h}, 24 \mathrm{~h}$ vs. $72 \mathrm{~h}$, or $48 \mathrm{~h}$ vs. $72 \mathrm{~h}$ and there was no commonality of the DEGs occurred between two of three time points (Fig. 1a, c).

The expression patterns of selected genes were confirmed by qPCR using the rice stem samples from the same batch of rice plants that were used for RNA-seq. A total of 20 genes were selected related to the signaling of phytohormones, primary metabolism, and secondary metabolism. The expression profiles of most genes tested by qPCR were consistent with those analyzed by RNA-seq although only one housekeeping gene was used in qPCR analysis (Fig. 2), which indicated the validation of the results from our transcriptome experiment.

\section{Series-cluster and enrichment analyses}

To refine the sets of genes that were differently expressed at a minimum of two time points, we used the STEM method, which is commonly used for the cluster of gene expression in transcriptomic studies [33]. The 4,729 DEGs were clustered into 26 possible model profiles (Fig. 3; Additional file 6: Table S6). Based on the expression dynamics of these DEGs, their expression patterns were assigned to five classes (Additional file 6: Table S6). Class I included 2,122 genes that showed a trend of up-regulated expression during the 72-h of larval feeding. Class II contained 1,318 genes showing a trend of down-regulated expression. Class III contained 873 genes 

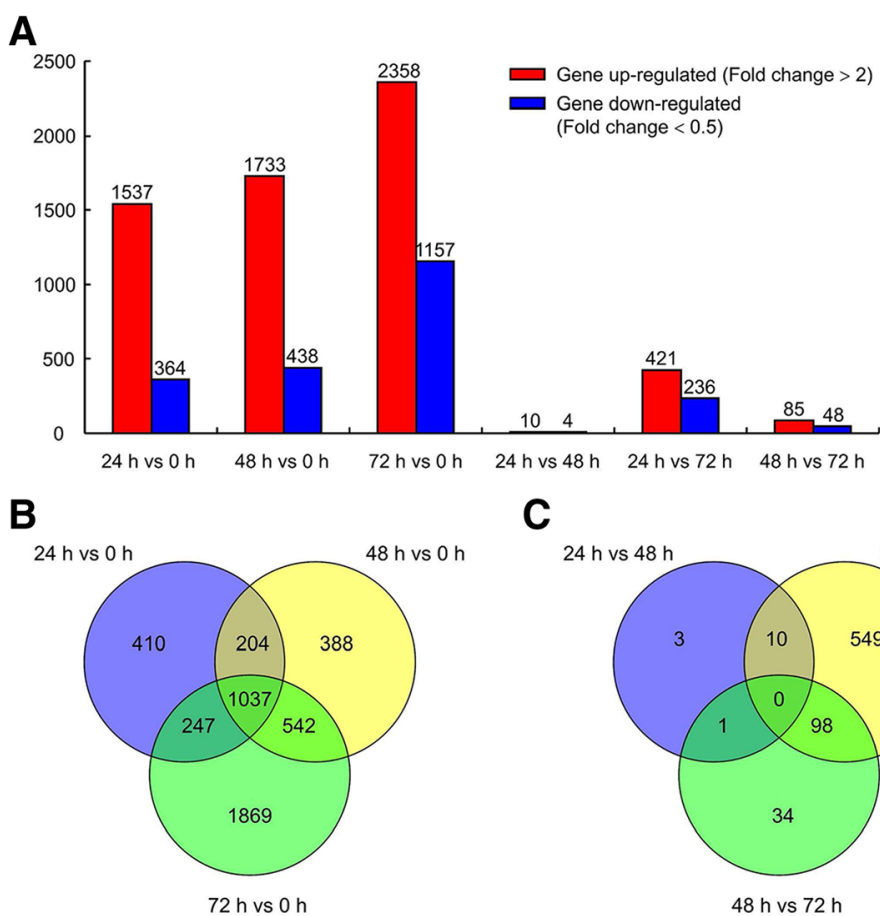

C

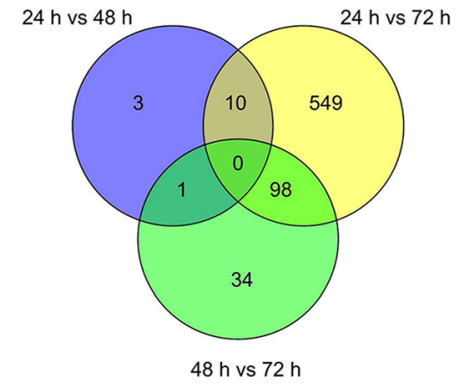

Fig. 1 Expression dynamics and comparative analyses of differentially expressed genes (DEGs) in rice plants damaged by Chilo suppressalis at different time points. a Bar graph of up- and down-regulated genes from pairwise comparisons (fold-change $>2$ or $<0.5$, and FDR $<0.05$ ). $\mathbf{b}, \mathbf{c}$ Veen diagram showing the common and uniquely regulated DEGs among different time points vs. control plants $(0 \mathrm{~h})(\mathbf{b})$ and among different time points (c)

that were up-regulated at early stage, but down-regulated at later stage. Class IV included 222 genes that were down-regulated at early stage but up-regulated at late stage. Class V contained the remaining 194 genes with irregular expression profile. GO analyses indicated that the number of significant GO terms with biological process categories in the five classes were $85,47,48,2$, and 5 , respectively (Additional file 8: Table S7). This indicates that most DEGs involved in the response to $C$. suppressalis damage contained in the first three classes. More details of the GO analyses for these DEGs are provided in Additional file 8: Table S7. Pathway enrichment analyses showed that genes in class I are mainly related to pathways of biosynthesis of plant secondary metabolites, plant hormone signal transduction, nitrogen metabolism, galactose, and terpenoid (Table 1). Genes in class II are mainly involved in primary metabolism such as nucleotide metabolism and photosynthesis, which may indicate the repressed activity of photosynthesis and the increased catabolism of nucleic acids. Genes in class III are mainly involved in pathways of biosynthesis of secondary metabolites including glucosinolate and phenylpropanoids and the metabolism of carbohydrates such as galactose, fructose, and mannose. The genes in class IV are mainly related to the metabolism of starch and sucrose, and to the biosynthesis of photosynthesis-antenna proteins, flavone, and flavonol. The genes in class $\mathrm{V}$ are mostly involved in secondary metabolism.

\section{Phytohormone-related DEGs}

A total of 9,221 Arabidopsis orthologs of rice genes were included in the Hormonometer analyses (Additional file 9: Table S8). Changes in gene expression induced by $C$. suppressalis in rice were positively correlated with those induced by SA (salicylic acid), JA (jasmonic acid), ABA (abscisic acid), and auxin treatments in Arabidopsis (Fig. 4). The changes in gene expression were negatively correlated with genes associated with cytokinin (CTK) signatures. These patterns were generally supported by $\mathrm{GO}$ analyses of the five classes (Additional file 8: Table S7).

\section{Transcription factors (TFs)-related DEGs}

Given the important regulatory function of TFs, we analyzed TFs-encoding genes by conducting a search of the Plant Transcription Factor Database (PlnTFDB,V3.0) (http://plntfdb.bio.uni-potsdam.de/v3.0/) [38]. We identified 385 TFs distributed in 39 families among the 4,729 DEGs (Additional file 10: Table S9). These TFs mainly include the following families: AP2-EREBP (apetala2ethylene-responsive element binding proteins) (50 genes), WRKY (37 genes), bHLH (basic helix-loop-helix) (27 


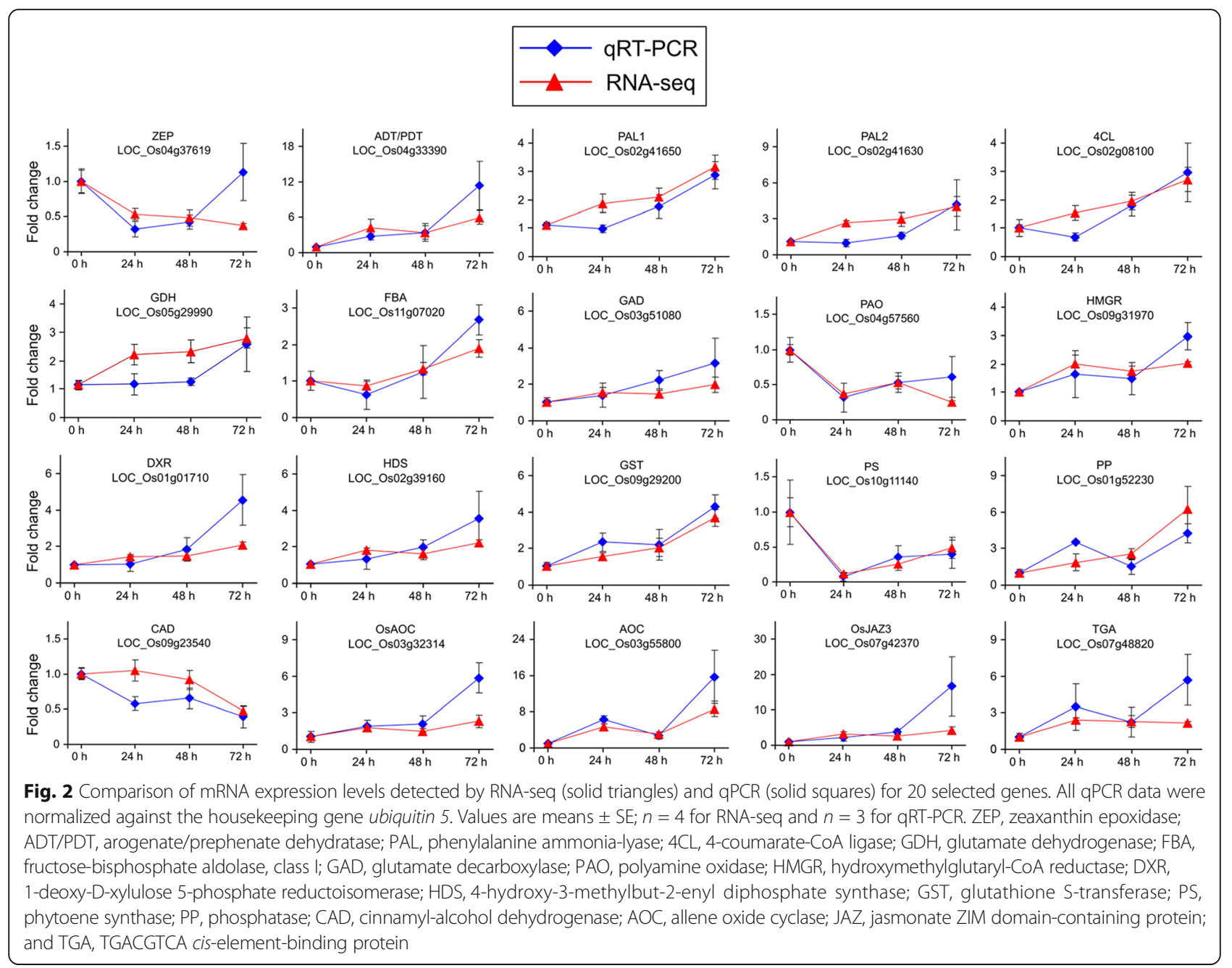

genes), MYB (myeloblastosis) (22 genes), NAC (NAM, ATAF1-2, and CUC2) (20 genes), Orphans (17 genes), HB (hunchback) (15 genes), MYB-related (13 genes), and bZIP (basic region/leucine zipper motif) (13 genes). Most of the genes belonging to AP2-EREBP, WRKY, MYB, bHLH, MYB-related, and NAC families are in class I. Half of the identified TFs from orphans and bZIP families are in class II. More details of the expression profiles of the identified TFs are provided in Additional file 10: Table S9.

\section{Metabolome composition analyses}

A total of 151 known metabolites were detected and quantified in rice plants during the $96 \mathrm{~h}$ of larval feeding (Additional file 11: Table S10). By mapping the general biochemical pathways based on KEGG and plant metabolic network (PMN), we divided the metabolites into seven classes, of which amino acids were the most prevalent (33\% of the metabolites), followed by carbohydrates (29\%) (Additional file 12: Figure S2). The secondary metabolites accounted for 7\% (Additional file 11: Table S10; Additional file 12: Figure S2).

\section{Integrated analyses of the transcriptomic and metabolic} data sets

Biosynthesis of aromatic amino acids, salicylic acid, and phenylpropanoids

The shikimate pathway is a major pathway in plants and is responsible for the biosynthesis of the aromatic amino acids Phe, Tyr, and Trp, as well as of auxin, SA, lignin, and phenylpropanoid [39]. Integration of the transcriptomic and metabolic data revealed that transcriptional up-regulation of the genes was accompanied by the elevation of the main metabolites in the pathways (Fig. 5; Additional file 13: Table S11). For example, all of the genes encoding the crucial enzymes in the shikimate pathway that accumulated throughout the $72 \mathrm{~h}$ of larval feeding belong to class I containing up-regulated DEGs (Fig. 5).

\section{Chilo suppressalis-induced changes in carbohydrate metabolism}

As products of photosynthesis, carbohydrates are the main source of stored energy in plants. Most DEGs involved in 

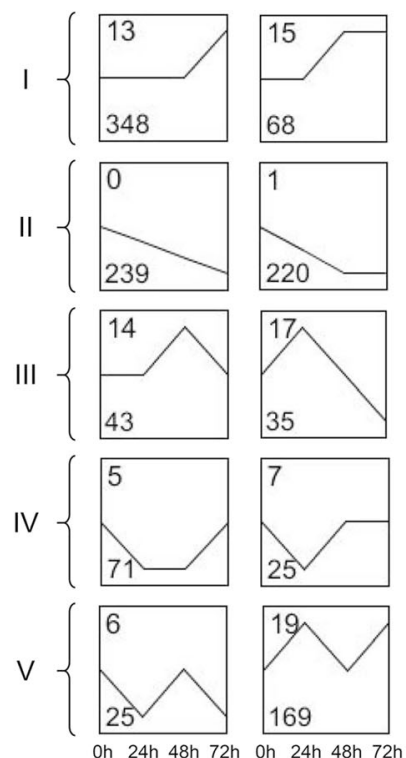
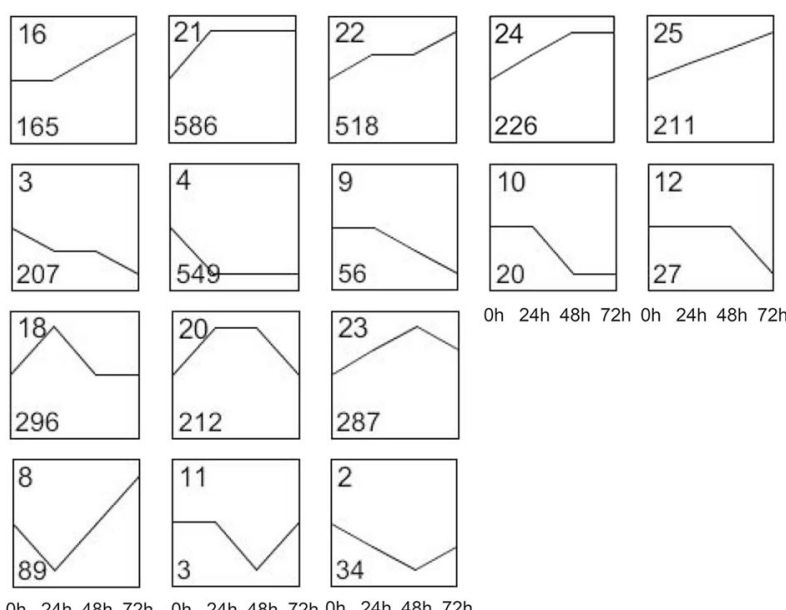

oh $24 \mathrm{~h} 48 \mathrm{~h} 72 \mathrm{~h}$ oh $24 \mathrm{~h} 48 \mathrm{~h} 72 \mathrm{~h}$ Oh $24 \mathrm{~h} 48 \mathrm{~h} 72 \mathrm{~h}$

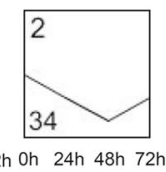

Fig. 3 Clustering and classification of 4,729 differentially expressed genes. The Roman numerals on the left indicate the class. The number in the top left corner in each panel indicates the identification number (ID) of the 26 profiles that were identified, and the number in the bottom left corner of each panel indicates the number of genes in the cluster

carbohydrate metabolism were up-regulated (Fig. 6b), with an exception of the genes encoding trehalose 6-phosphate synthase (TPS) and 4-alpha-glucanotransferase (AGLS). Consistently, metabolic analysis showed that except for oligosaccharides and galactinol, all monosaccharides (orbitol, galactitol, glucose, fructose, and xylose) increased over time (Fig. 6c; Additional file 11: Table S10).

\section{Effects of Chilo Suppressalis feeding on amino acids, organic acids, and nitrogen metabolism}

Our analyses showed that genes encoding enzymes such as glutamate decarboxylase (GAD), N-carbamoylputrescine amidase (CPA), ornithine decarboxylase (ODC), and Laspartate oxidase (LASPO) were up-regulated; while those encoding adenylosuccinate lyase (ASL), and delta-1-pyrroline-5-carboxylate synthetase (P5CS) were down-regulated over time. As expected, the contents of metabolites ornithine, gamma-aminobutyrate and putrescine increased, while the levels of aspartate and spermidine decreased in rice plants during C. suppressalis feeding due to action of the enzymes mentioned above (Fig. 7a, b). In addition, we also detected increased levels of other amino acids such as Pro, Ala, and Asn (Fig. 7c).

\section{Chilo suppressalis-induced changes in terpenoid metabolism}

The analysis was focused on the genes that participate in terpenoid metabolism (Fig. 8; Additional file 13: Table S11). The four genes that encode the following crucial enzymes in the methylerythritol phosphate
(MEP) pathway were up-regulated by C. suppressalis feeding: 1-deoxy-D-xylulose 5-phosphate synthase (DXS), 1-deoxy-D-xylulose 5-phosphate reductoisomerase (DXR), 4-diphosphocytidyl-2-C-methyl-D-erythritol kinase (MCT), and 4-hydroxy-3-methylbut-2-enyl diphosphate synthase (HDS). In addition, the gene encoding hydroxymethylglutaryl-CoA reductase (HMGR) and genes encoding geranyl diphosphate synthase (GPS), farnesyl diphosphate synthase (FPS), and geranylgeranyl diphosphate synthase (GGPS) were also up-regulated induced by $C$. suppressalis feeding. The expression of several genes encoding enzymes in the diterpenoid biosynthesis and carotenoid biosynthesis pathways were also altered by C. suppressalis feeding. Of these genes, 9-cis-epoxycarotenoid dioxygenase (NCED) were substantially up-regulated. In contrast, the genes encoding GA 2-oxidase (GA2o) and zeaxanthin epoxidase (ZEP) were down-regulated throughout the larval feeding period.

\section{Discussion}

The current study describes the first effort to combine transcriptomic and metabolic techniques for the comparative analyses of the genes and the metabolites involved in rice plant responses to damage caused by $C$. suppressalis larvae. The results increase our understanding of the mechanisms underlying the dynamic responses of rice plants to caterpillar feeding.

Gene expression analyses revealed that more DEGs were up-regulated than down-regulated in response to feeding by $C$. suppressalis larvae. This is consistent with 
Table 1 Summary of significantly enriched $(P<0.05)$ pathway terms associated with differentially expressed genes (DEGs)

\begin{tabular}{|c|c|c|c|c|}
\hline Class $^{a}$ & Pathway ID & Pathway term & Number of DEGs & $P$ value* \\
\hline \multirow[t]{19}{*}{ I } & PATH:01110 & Biosynthesis of secondary metabolites & 136 & 2.03E-05 \\
\hline & PATH:00940 & Phenylpropanoid biosynthesis & 37 & 4.43E-05 \\
\hline & PATH:00910 & Nitrogen metabolism & 13 & $2.65 \mathrm{E}-04$ \\
\hline & PATH:00592 & alpha-linolenic acid metabolism & 13 & $3.56 \mathrm{E}-04$ \\
\hline & PATH:04075 & Plant hormone signal transduction & 33 & $3.64 \mathrm{E}-04$ \\
\hline & PATH:00062 & Fatty acid elongation & 11 & $1.09 \mathrm{E}-03$ \\
\hline & PATH:00945 & Stilbenoid, diarylheptanoid, and gingerol biosynthesis & 19 & $1.32 \mathrm{E}-03$ \\
\hline & PATH:00360 & Phenylalanine metabolism & 26 & $1.50 \mathrm{E}-03$ \\
\hline & PATH:01100 & Metabolic pathways & 180 & $1.98 \mathrm{E}-03$ \\
\hline & PATH:00941 & Flavonoid biosynthesis & 15 & $2.68 \mathrm{E}-03$ \\
\hline & PATH:04626 & Plant-pathogen interaction & 40 & $3.24 \mathrm{E}-03$ \\
\hline & PATH:00280 & Valine, leucine and isoleucine degradation & 10 & $3.70 \mathrm{E}-03$ \\
\hline & PATH:00052 & Galactose metabolism & 11 & 4.30E-03 \\
\hline & PATH:00903 & Limonene and pinene degradation & 15 & $5.76 \mathrm{E}-03$ \\
\hline & PATH:00480 & Glutathione metabolism & 17 & $8.59 \mathrm{E}-03$ \\
\hline & PATH:00561 & Glycerolipid metabolism & 11 & $8.75 \mathrm{E}-03$ \\
\hline & PATH:00410 & beta-alanine metabolism & 7 & $2.00 \mathrm{E}-02$ \\
\hline & PATH:00900 & Terpenoid backbone biosynthesis & 9 & $2.43 \mathrm{E}-02$ \\
\hline & PATH:00760 & Nicotinate and nicotinamide metabolism & 4 & 4.37E-02 \\
\hline \multirow[t]{11}{*}{$\|$} & PATH:03008 & Ribosome biogenesis in eukaryotes & 31 & $2.77 \mathrm{E}-14$ \\
\hline & PATH:03010 & Ribosome & 41 & 1.40E-08 \\
\hline & PATH:00196 & Photosynthesis - antenna proteins & 10 & $1.20 \mathrm{E}-07$ \\
\hline & PATH:00230 & Purine metabolism & 19 & $1.24 \mathrm{E}-03$ \\
\hline & PATH:00240 & Pyrimidine metabolism & 16 & 2.67E-03 \\
\hline & PATH:03013 & RNA transport & 19 & $3.63 \mathrm{E}-03$ \\
\hline & PATH:03018 & RNA degradation & 13 & $8.68 \mathrm{E}-03$ \\
\hline & PATH:03410 & Base excision repair & 7 & $1.31 \mathrm{E}-02$ \\
\hline & PATH:03450 & Non-homologous end-joining & 3 & $1.74 \mathrm{E}-02$ \\
\hline & PATH:03440 & Homologous recombination & 7 & 3.87E-02 \\
\hline & PATH:03020 & RNA polymerase & 6 & $4.08 \mathrm{E}-02$ \\
\hline \multirow[t]{14}{*}{ III } & PATH:01110 & Biosynthesis of secondary metabolites & 89 & $2.05 \mathrm{E}-13$ \\
\hline & PATH:00940 & Phenylpropanoid biosynthesis & 26 & $2.69 \mathrm{E}-07$ \\
\hline & PATH:00010 & Glycolysis/Gluconeogenesis & 17 & $5.30 \mathrm{E}-06$ \\
\hline & PATH:00360 & Phenylalanine metabolism & 20 & $6.99 \mathrm{E}-06$ \\
\hline & PATH:00520 & Amino sugar and nucleotide sugar metabolism & 18 & $1.12 \mathrm{E}-05$ \\
\hline & PATH:00966 & Glucosinolate biosynthesis & 4 & $7.22 \mathrm{E}-04$ \\
\hline & PATH:00380 & Tryptophan metabolism & 7 & $1.19 \mathrm{E}-03$ \\
\hline & PATH:01100 & Metabolic pathways & 89 & $2.00 \mathrm{E}-03$ \\
\hline & PATH:00909 & Sesquiterpenoid and triterpenoid biosynthesis & 4 & 4.89E-03 \\
\hline & PATH:00051 & Fructose and mannose metabolism & 7 & $8.44 \mathrm{E}-03$ \\
\hline & PATH:00904 & Diterpenoid biosynthesis & 5 & $8.62 \mathrm{E}-03$ \\
\hline & PATH:00052 & Galactose metabolism & 6 & $1.54 \mathrm{E}-02$ \\
\hline & PATH:00030 & Pentose phosphate pathway & 5 & $3.29 \mathrm{E}-02$ \\
\hline & PATH:00591 & Linoleic acid metabolism & 3 & 4.14E-02 \\
\hline
\end{tabular}


Table 1 Summary of significantly enriched $(P<0.05)$ pathway terms associated with differentially expressed genes (DEGs) (Continued)

\begin{tabular}{|c|c|c|c|c|}
\hline & PATH:00944 & Flavone and flavonol biosynthesis & 3 & 4.62E-02 \\
\hline \multirow[t]{3}{*}{ IV } & PATH:00500 & Starch and sucrose metabolism & 6 & 2.24E-03 \\
\hline & PATH:00196 & Photosynthesis - antenna proteins & 2 & 4.87E-03 \\
\hline & PATH:00944 & Flavone and flavonol biosynthesis & 2 & 1.23E-02 \\
\hline \multirow[t]{9}{*}{ V } & PATH:01110 & Biosynthesis of secondary metabolites & 17 & $2.24 \mathrm{E}-03$ \\
\hline & PATH:01100 & Metabolic pathways & 22 & 4.03E-03 \\
\hline & PATH:00940 & Phenylpropanoid biosynthesis & 6 & $6.04 \mathrm{E}-03$ \\
\hline & PATH:00500 & Starch and sucrose metabolism & 5 & $9.00 \mathrm{E}-03$ \\
\hline & PATH:00944 & Flavone and flavonol biosynthesis & 2 & 1.10E-02 \\
\hline & PATH:00902 & Monoterpenoid biosynthesis & 1 & 2.00E-02 \\
\hline & PATH:00941 & Flavonoid biosynthesis & 3 & $2.31 \mathrm{E}-02$ \\
\hline & PATH:00460 & Cyanoamino acid metabolism & 2 & $3.62 \mathrm{E}-02$ \\
\hline & PATH:01110 & Biosynthesis of secondary metabolites & 17 & $2.24 \mathrm{E}-03$ \\
\hline
\end{tabular}

${ }^{\mathrm{a} C l a s s}$ numbers refer to Fig. 3

* $P$ values for modified Fisher's exact test

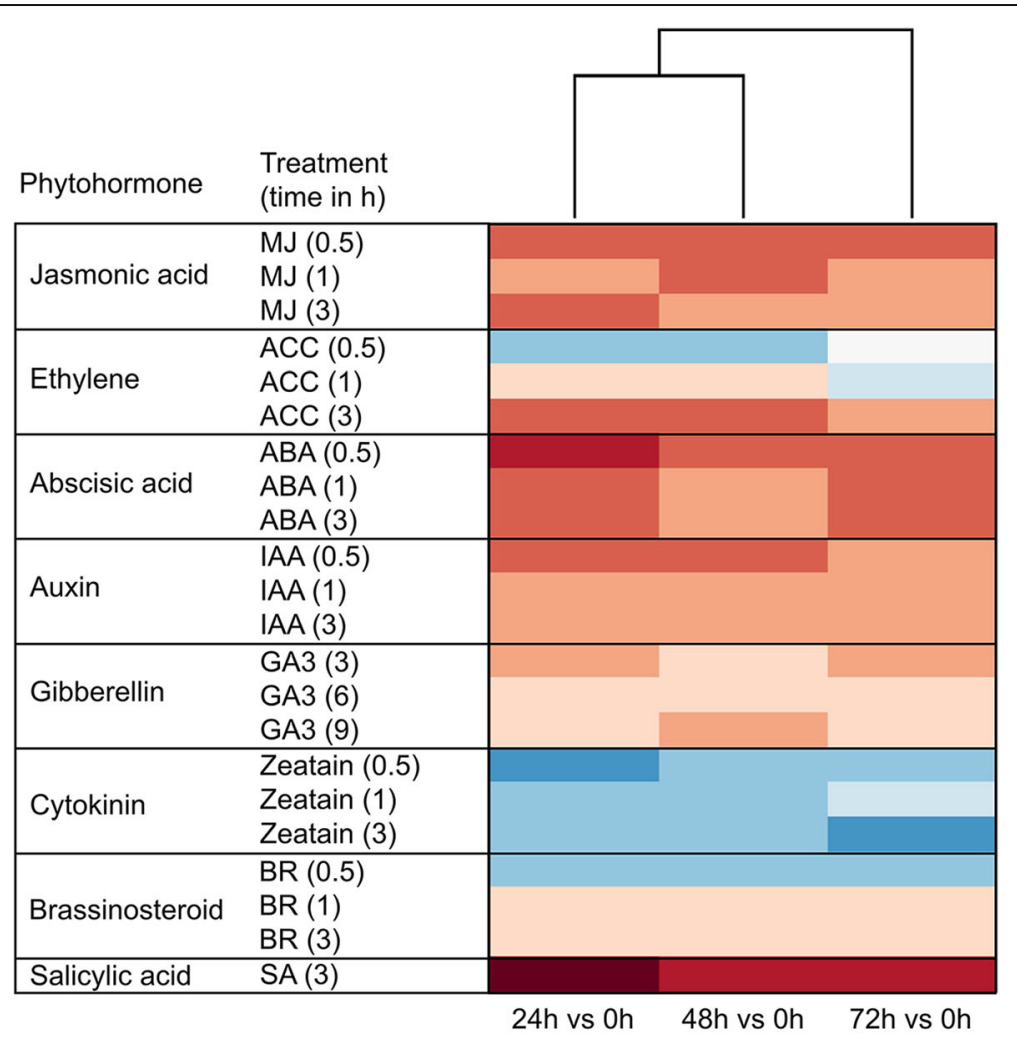

Correlation values

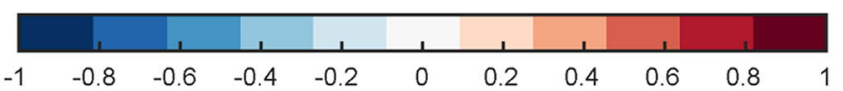

Fig. 4 Hormonometer analysis of differential gene expression in rice in response to Chilo suppressalis feeding. The response in gene expression in rice to Chilo suppressalis feeding (for $0,24,48$, or 72 h) treatments was compared with that of Arabidopsis at 30, 60, and 180 min, or 3, 6, and $9 \mathrm{~h}$ after hormone application. Red shading indicates a positive correlation between the rice response to a C. suppressalis treatment and the Arabidopsis response to a hormone treatment; blue shading indicates a negative correlation. MJ, methyl jasmonate; ACC, 1-aminocyclopropane-1-caroxylic acid (a metabolic precursor of ethylene); ABA, abscisic acid; IAA, indole-3-acetic acid; GA3, gibberellic acid 3; BR, brassinosteroid; and SA, salicylic acid 


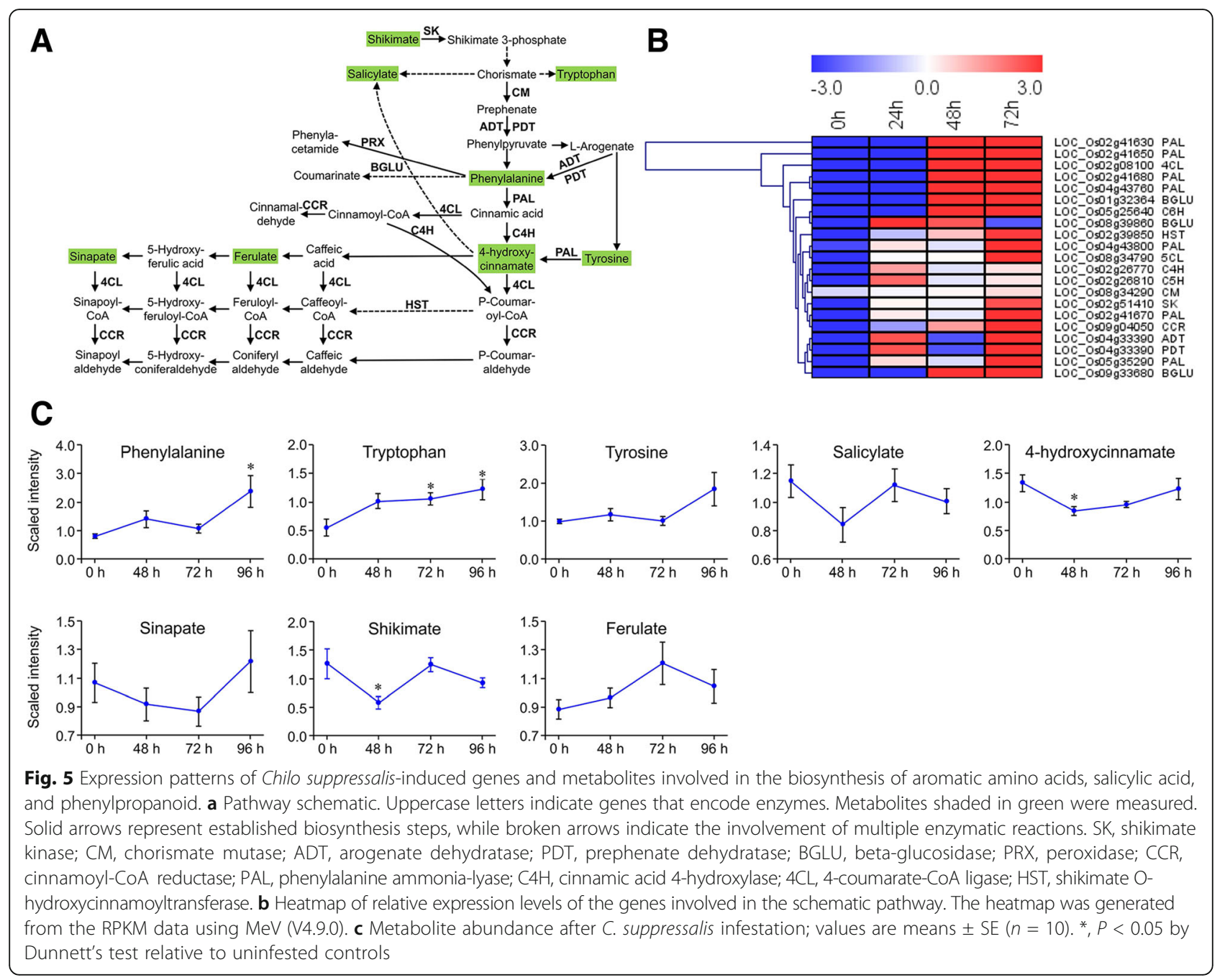

previous findings concerning aphid-infested maize [7] and maize that was mechanically wounded and then treated with the oral secretions of Mythimna separata [9]. Similarly, more DEGs were up-regulated than downregulated when Arabidopsis plants were individually infested with Myzus persicae, Brevicoryne brassicae, Spodoptera exigua, or Pieris rapae [40], or when cotton was damaged by the chewing insects Helicoverpa armigera or Anthonomus grandis [41]. However, there were also studies reporting that more DEGs were down-regulated than up-regulated, or the numbers of up- and down-regulated DEGs were equivalent when rice plants were damaged by C. suppressalis [8] or the brown planthopper $N$. lugens $[42,43]$, or when cotton plants were infested with the whitefly Bemisia tabaci or the aphid Aphis gossypii $[6,44]$. This variability might be explained by differences in herbivore species, plant species, plant tissues infested, the duration of infestation, and the techniques used for the detection of gene expression [40].
As the key regulators of transcription, TFs are important in plant responses to herbivory [5, 8, 45-47]. In our transcriptome analyses, we identified $385 \mathrm{TF}$ genes that responded to $C$. suppressalis feeding, suggesting that the induced defense response is complex and involves a substantial change in rice metabolism. The TF families whose expression was most altered by $C$. suppressalis feeding were AP2-EREBP and WRKY. Evidence increasingly indicates that WRKYs play significant roles in plant development and in responses to biotic and abiotic stresses [5, 8, 45-47], and members of the AP2-EREBP family mediate defense against biotic and/or abiotic stress [45]. For example, it was recently found that OsWRKY7O mediates the prioritization of defense over growth by positively regulating cross-talk between JA and SA when rice is attack by C. suppressalis [47], and OsWRKY53 is a negative regulator of plant growth and an early suppressor of induced defenses [46], both of which belong to WRKY family. The function of TFs in 


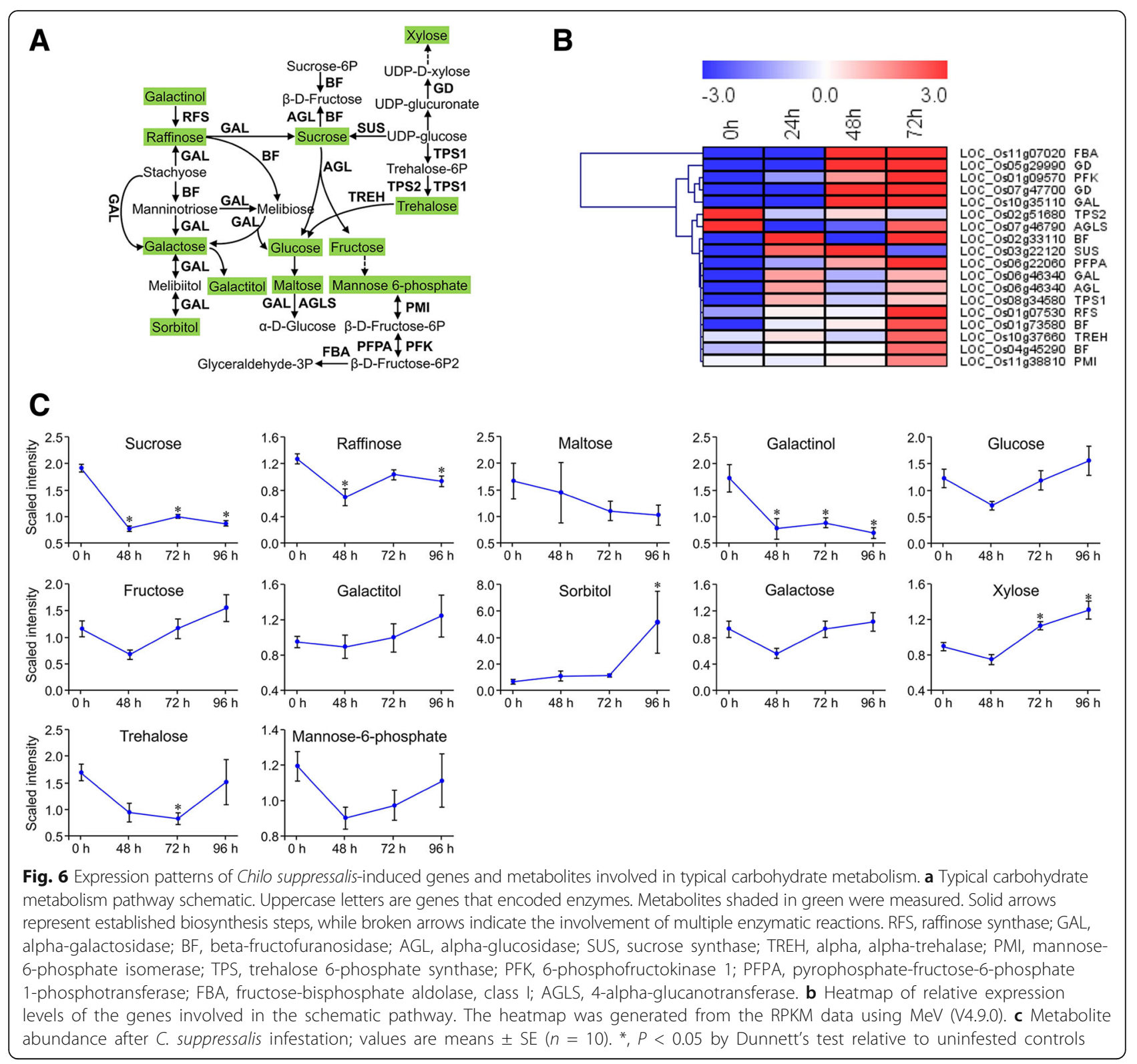

the defense of rice against insects warrants further research.

Phytohormones play important roles in a complex regulatory network that is essential for herbivore-induced response as previously reported $[1,4,48]$ and as also indicated by our Hormonometer analysis. Our results showed that $C$. suppressalis elicited the expression of genes associated with JA and SA, which is consistent with a previous study [8]. In turn, exogenous application of methyl JA or JA to rice plants reduced the performance of two root herbivores, the cucumber beetle Diabrotica balteata and the rice water weevil Lissorhoptrus oryzophilus [49], and induced the release of volatiles that attract parasitoids [50]. SA, which is a central phytohormone in the shikimate pathway, plays an importance role in the defense against biotrophic pathogens and piercing/sucking insects [1]. Our data showed that a number of rice SArelated genes were up-regulated by $C$. suppressalis larval feeding (Fig. 5b). Although studies have reported that crosstalk between JA and SA is negative in Arabidopsis [51], and that JA-dependent defense may be hampered by SA and vice versa $[5,19]$, our findings are consistent with the evidence that SA and JA can have overlapping or even synergistic effects in rice $[8,51]$.

We found that changes in gene expression induced by C. suppressalis in rice were positively correlated with changes induced by ABA treatment in Arabidopsis, which agrees with previous results in several plant-insect systems $[5,7,9,40,44]$. The role of ABA in regulating defense against pathogens in rice has been well documented [51], 


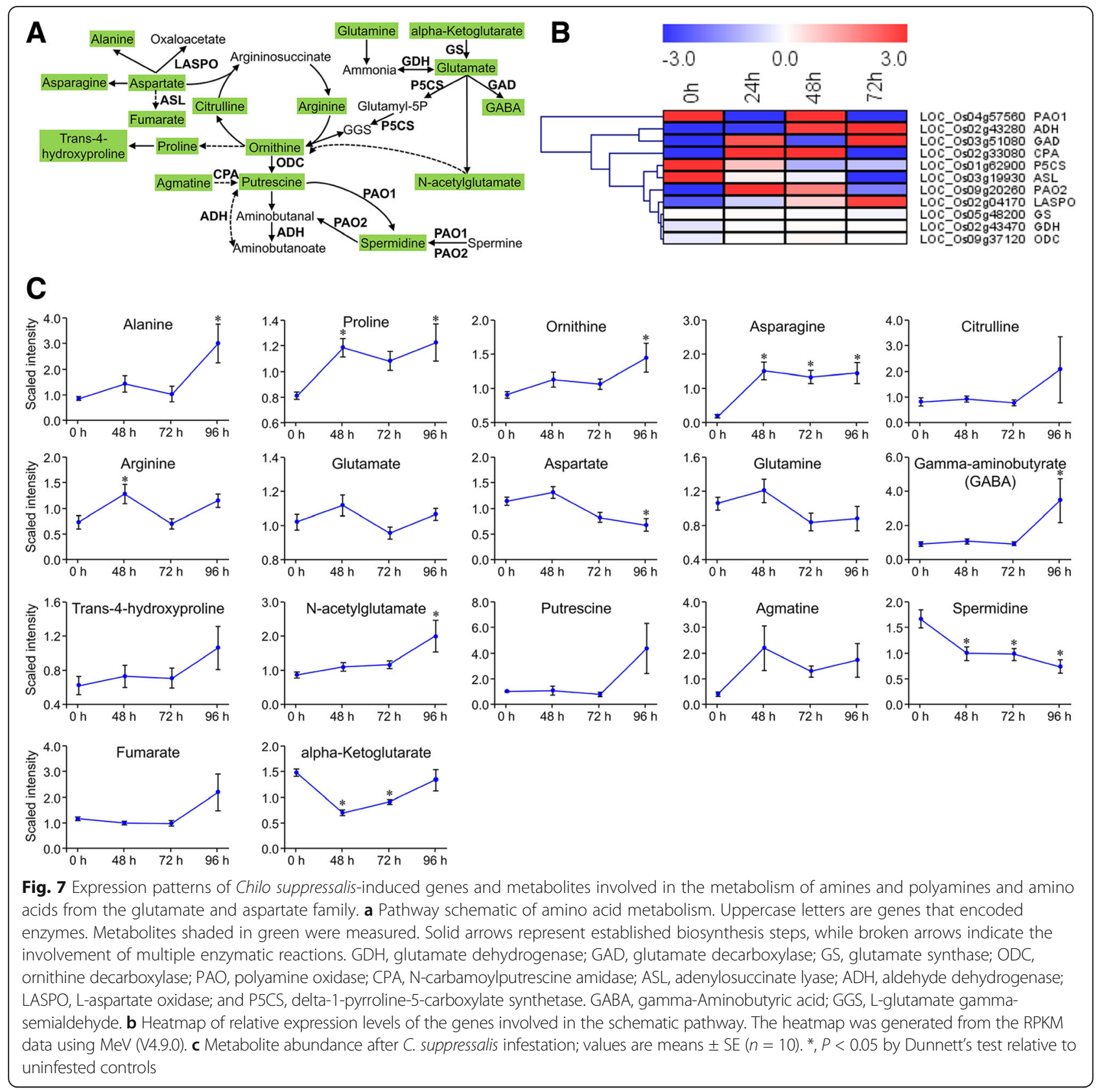

but its role in resistance to insects is much less understood. Our results suggest that ABA signature may also play a vital role in rice defense against insect herbivores, although researchers recently reported that applying $\mathrm{ABA}$ to rice roots did not affect the performance of $D$. balteata and L. oryzophilus [49]. We supposed that ABA may function in other ways in rice plant defense against herbivory, but further studies are needed for clarifying this hypothesis. In contrast, we found a negative correlation between CTK-induced and C. suppressalis-induced gene expression (Fig. 4). This negative correlation, which has been also observed in other plant species [7, 34, 52], may reflect the decrease in growth rate of rice plants caused by $C$. suppressalis infestation.

Insect infestation causes many changes in both primary and secondary metabolism, and the reconfiguration of metabolism is a common defense strategy [11, 48, 53]. Our MapMan analyses and GO and pathway enrichment analyses indicate that rice plants reprogram both primary and secondary metabolism in response to C. suppressalis feeding (Table 1; Additional file 7: Figure S1 and Additional file 8: Table S7). Reductions in photosynthesis, as indicated by down-regulation of photosynthesis-related genes, is a common response to 


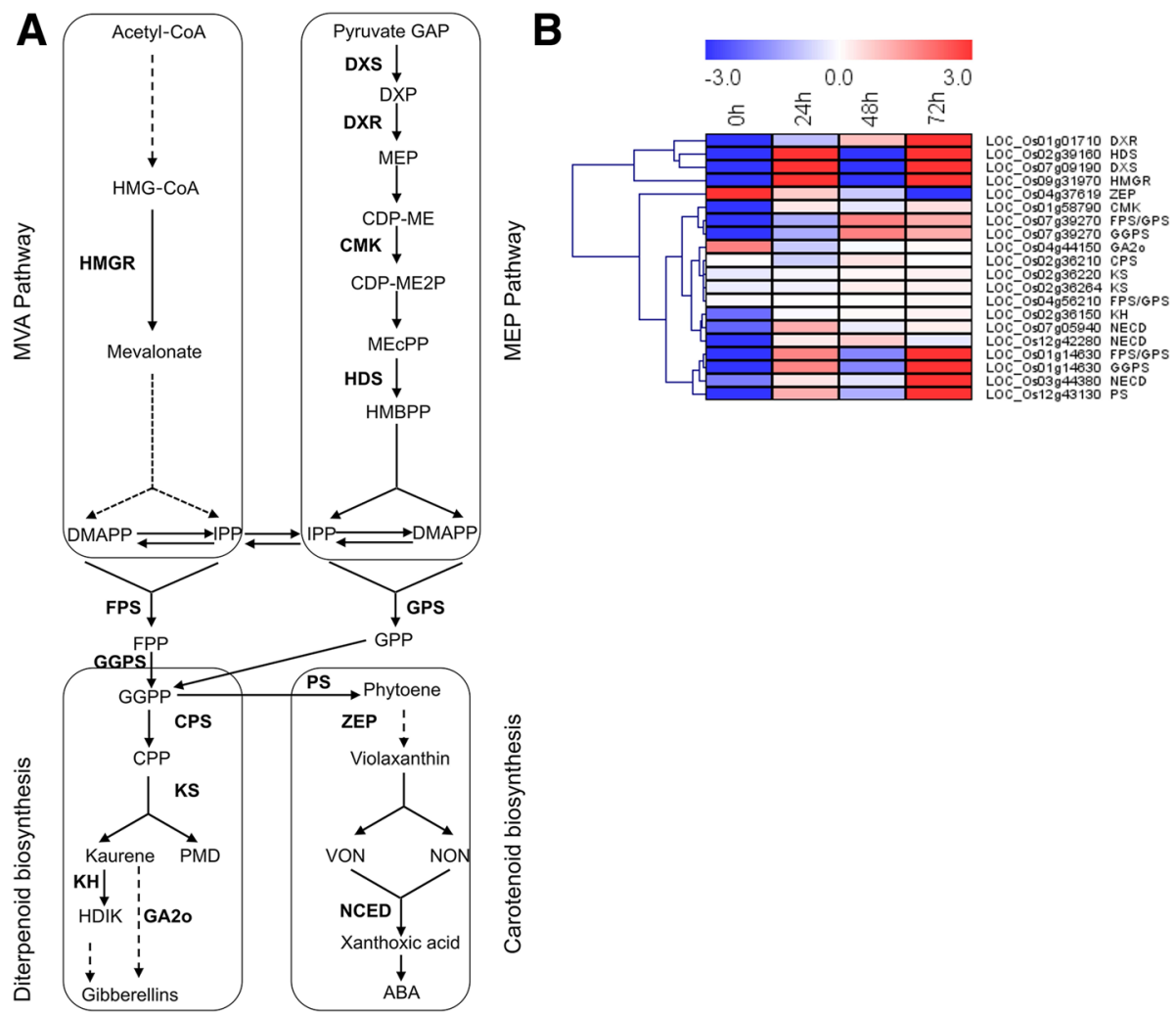

Fig. 8 Expression patterns of Chilo suppressalis-induced genes involved in terpenoid biosynthetic pathways. a Pathway schematic of terpenoid metabolism. Uppercase letters are genes that encoded enzymes. Solid arrows represent established biosynthesis steps, while broken arrows indicate the involvement of multiple enzymatic reactions. MVA, mevalonate; MEP, 2-C-methyl-D-erythritol 4-phosphate; HMG-CoA, Hydroxymethylglutaryl-CoA; HMGR, HMG-CoA reductase; DMAPP, dimethylallyl pyrophosphate; IPP, isopentenyl pyrophosphate; IDI, IPP isomerase; GAP, glyceraldehyde-3-phosphate; DXP, 1-deoxy-D-xylulose 5-phosphate; DXS, DXP synthase; DXR, 1-deoxy-D-xylulose 5-phosphate reductoisomerase; CDP-ME, 4-diphosphocytidyl-2-C-methyl-D-erythritol; MCT, 4-diphosphocytidyl-2-C-methyl-Derythritol synthase; CMK, 4-diphosphocytidyl-2-Cmethyl-D-erythritol kinase; CDP-ME-2P, 4-diphosphocytidyl-2-C-methyl-D-erythritol 2-phosphate; MECPP, 2-C-methyl-D-erythritol 2,4-cyclodiphosphate; HDS, 4-hydroxy-3-methylbut-2-enyl diphosphate synthase; HMBPP, 4-hydroxy-3-methylbut-2-enyl diphosphate; GPP, geranyl diphosphate; GPS, GPP synthase; FPP, farnesyl diphosphate; FPS, FPP synthase; GGPP, geranylgeranyl diphosphate; GGPS, GGPP synthase; CPP, copalyl diphosphate; CPS, CPP synthase; KS, kaurene synthase; PMD, Pimara-8(14),15-diene; KH, Ent-isokaurene C2-hydroxylase; HDIK, ent-2-alpha-Hydroxyisokaurene; GA20, GA 2-oxidase; PSY, phytoene synthase; PS, phytoene synthase; ZEP, zeaxanthin epoxidase; VON, 9-cis-Violaxanthin; NON, 9'-cis-Neoxanthin; NCED, 9-cis-epoxycarotenoid dioxygenase; ABA, abscisic acid. $\mathbf{b}$ Heatmap of relative expression levels of the genes involved in the schematic pathway. The heatmap was generated from the RPKM data using MeV (V4.9.0)

insect feeding $[5,8,11,40,53]$ what was also confirmed in the current study. The down-regulation of photosynthetic genes accompanied by the up-regulation of defenserelated genes may allow rice plants to redirect resources toward defense.

Photosynthesis is reduced in insect-attacked plants, while plants require energy and carbon to produce defense-related metabolites [11, 53]. Many plant species respond to the damage by promoting the catabolism of energy storage compounds, as can be reflected by the increased activity of invertase and the increased expression of genes encoding enzymes that catalyze the degradation of complex carbohydrates [11]; such changes were also evident in the current study. For example, we found that genes encoding invertases such as alpha-glucosidase $(A G L)$, beta-fructofuranosidase $(B F)$, and alpha-galactosidase $(G A L)$ were up-regulated in response to $C$. suppressalis feeding. As a result, the contents of oligosaccharides, raffinose, and galattinol declined while those of monosaccharides increased (Fig. 6c). As the major form of nitrogen in plants, amino acids are the major growthlimiting nutrients for herbivores and are also precursors for the production of defense-related metabolites. Amino acids are therefore important in the interactions between plants and herbivores [11]. Our metabolic analyses showed that the contents of most amino acids were increased by C. suppressalis feeding (Figs. 5 and 7 and Additional file 11: Table S10). Among these amino acids, Tryptophan (Trp), for instance, was significantly increased by C. suppressalis feeding (Fig. 5c). Trp can 
serve as a precursor for defensive metabolites. Similar results were also reported by previous studies $[40,49]$. Phe is a precursor for shikimate-mediated biosynthesis of phenylpropanoids [39]. Our results showed the increased phenylalanine ammonia-lyase $(P A L)$ gene expression was accompanied by the elevated levels of Phe over time. This was in consent with the previous study by Liu et al. [54], in which both activated PAL gene expression and increased Phe levels were detected in rice plants that had damaged by $N$. lugens. Another important amino acid, gamma-aminobutyric acid (GABA) also increased in content at later stage when rice plants were fed by $C$. suppressalis larvae. Similar results were found when rice plants were fed by $N$. lugens [54]. Consistent results were reported that feeding by $S$. littoralis larvae causes the accumulation of GABA in leaves of Arabidopsis, and this accumulation reduces insect feeding [55]. The role of GABA in rice defense against herbivores requires further investigation. Although herbivore-induced accumulation of amino acids can support the production of defensive metabolites, the accumulation of amino acids might also benefit the herbivore [1, 7]. In support of the latter inference, we observed that the rice brown planthopper $N$. lugens was more attracted to rice plants infested with $C$. suppressalis than to uninfested plants (Wang et al., unpublished data).

In plants, secondary metabolites play an important role in the defense response to insect feeding. Phenylpropanoids which are mainly biosynthesised through the shikimate pathway, have been widely reported to be induced by insect feeding serving as direct resistance to herbivory $[5,12]$. In the current study, we found that genes involved in the shikimate pathway such as shikimate kinase (SK), chorismate mutase $(C M)$, arogenate dehydratase $(A D T)$, prephenate dehydratase (PDT), phenylalanine ammonialyase $(P A L)$, and cinnamic acid 4-hydroxylase $(C 4 H)$ were induced and phenylpropanoids such as 4hydroxycinnamate and ferulate were accumulated as a response to attack by $C$. suppressalis. These results suggest that the shikimate-mediated secondary metabolism was vitally important for rice defense against $C$. suppressalis larval feeding. Terpenoids, which are the most common group of secondary metabolites, can directly affect insect performance or indirectly attract natural enemies of the attacking herbivore $[1,4,56,57]$. In plants, all terpenoids are derived from the mevalonic acid (MVA) pathway and the methylerythritol phosphate (MEP) pathway [58]. In rice, infestation by chewing herbivores, such as $C$. suppressalis, S. frugiperda, or Cnaphalocrocis medinalis induces the release of a complex of blend of volatiles that increase the search efficiency of natural enemies [14]. In the current work, the expression of HMGR, which is the critical regulator that catalyzes the conversion of HMG-CoA to mevalonate in the MVA pathway [58], was up- regulated by $C$. suppressalis feeding. Farnesyl diphosphate (FPP), geranyl diphosphate (GPP) and geranylgeranyl diphosphate (GGPP) are the main precursors in the biosynthesis of monoterpenes, sesquiterpenes and triterpenes, and diterpenes [58]. Genes encoding enzymes that catalyze dimethylallyl pyrophosphate (DMAPP)/isopentenyl pyrophosphate (IPP) into FPP or GPP and that catalyze FPP to GGPP were also found to be up-regulated in our study. Moreover, key genes involved in the diterpenoid and carotenoid pathways were also activated by C. suppressalis feeding (Fig. 8). Previous studies have shown that rice plants damaged by $C$. suppressalis for at least $24 \mathrm{~h}$ increased their release of the terpenes as limonene, copaene, $\beta$-caryophyllene, $\alpha$ bergamotene, germacrene $\mathrm{D}, \delta$-selinene, and $\alpha$-cedrene $[8,57]$.

\section{Conclusions}

In summary, our integrated transcriptome and metabolome analyses generated a large data set concerning the dynamic defense of rice plants induced by $C$. suppressalis attack. The defense responses involved primary metabolisms, including photosynthesis, amino acid metabolism, and carbohydrate metabolism, and secondary metabolisms, including the biosynthesis of phenylpropanoids and terpenoids. The genes and metabolic networks identified in this study provide new insights into rice defense mechanisms and the current findings will provide clues for the development of insect-resistant rice cultivars as has for example been reported for soybeans with resistance to nematodes [59-61].

\section{Additional files}

Additional file 1: Table S1. Genes and primer pairs used for quantitative real-time PCR. (XLS 34 kb)

Additional file 2: Table S2. Summary of RNA sequencing and mapping using the rice genome (Oryza sativa) as reference. (XLS 29 kb)

Additional file 3: Table S3. Summary of gene structures. (XLS 31 kb)

Additional file 4: Table S4. Genes detected in all samples. (XLS 14574 kb)

Additional file 5: Table S5. All differentially expressed genes between any two groups. (XLS $1102 \mathrm{~kb}$ )

Additional file 6: Table S6. Five classes of the differentially expressed genes. (XLS $342 \mathrm{~kb}$ )

Additional file :7 Figure S1. Comparisons of metabolic changes in rice plants that had been fed by Chilo suppressalis larvae for different durations. (a) $24 \mathrm{~h}$ vs $0 \mathrm{~h}$. (b) $48 \mathrm{~h}$ vs $0 \mathrm{~h}$. (C) $72 \mathrm{~h}$ vs $0 \mathrm{~h}$. The colour intensity indicates the expression ratio at logarithmic scale (red: up-regulated, blue: downregulated). (TIF $1806 \mathrm{~kb}$ )

Additional file 8: Table S7. Significant (FDR < 0.01) GO terms (biological processes) associated with the grouped DEGs. (XLS 54 kb)

Additional file 9: Table S8. Orthologous Arabidopsis and rice genes used for Hormonometer analysis. (XLS 2918 kb)

Additional file 10: Table S9. The list of Chilo suppressalis-responsive transcription factors (TFs). (XLS 61 kb) 
Additional file 11: Table S10. Metabolic profiles for Chilo Suppressalis damaged rice plants $(0,48,72$ and $96 \mathrm{~h}$ after infection). (XLS $103 \mathrm{~kb})$

Additional file 12: Figure S2. Functional categorization of 151 rice metabolites across the four time points. (TIF $377 \mathrm{~kb}$ )

Additional file 13: Table S11. Genes derived from RNA-seq involved in metabolism based on KEGG pathway maps. (XLS 33 kb)

\section{Abbreviations}

4CL: 4-coumarate-CoA ligase; ABA: Abscisic acid; ACC: 1-aminocyclopropane1-caroxylic acid (a metabolic precursor of ethylene); $\mathrm{ADH}$ : Aldehyde dehydrogenase; ADT/PDT: Arogenate/prephenate dehydratase; AGl: Arabidopsis gene identifies; AGL: Asalpha-glucosidase; AGLS: 4-alphaglucanotransferase; AOC: Allene oxide cyclase; AP2-EREBP: Apetala2-ethyleneresponsive element binding proteins; ASL: Adenylosuccinate lyase; BF: Betafructofuranosidase; BGLU: Beta-glucosidase; bHLH: Basic helix-loop-helix: BR: Brassinosteroid; bZIP: Basic region/leucine zipper motif; $\mathrm{C} 4 \mathrm{H}$ : Cinnamic acid 4-hydroxylase; CAD: Cinnamyl-alcohol dehydrogenase; CCR: CinnamoylCoA reductase; CDP-ME: 4-diphosphocytidyl-2-C-methyl-D-erythritol; CDPME-2P: 4-diphosphocytidyl-2-C-methyl-D-erythritol 2-phosphate; CM: Chorismate mutase; CMK: 4-diphosphocytidyl-2-C-methyl-D-erythritol kinase; CPA: N-carbamoylputrescine amidase; CPP: Copalyl diphosphate: CPS: CPP synthase; CTK: Cytokinin; DEG: Differentially expressed genes; DMAPP: Catalyze dimethylallyl pyrophosphate; DXP: 1-deoxy-D-xylulose 5phosphate; DXR: 1-deoxy-d-xylulose 5-phosphate reductoisomerase; DXS: 1deoxy-d-xylulose 5-phosphate synthase; FBA: Fructose-bisphosphate aldolase, class I; FDR: False discovery rate; FPP: Farnesyl diphosphate; FPS: Farnesyl diphosphate synthase; GA20: GA 2-oxidase; GA3: Gibberellic acid 3; GABA: Gamma-aminobutyric acid; GAD: Glutamate decarboxylase; GAL: Andalpha-galactosidase; GAP: Glyceraldehyde-3-phosphate; GC-MS: Gas chromatography-mass spectrometry; GDH: Glutamate dehydrogenase; GGPP: Geranylgeranyl diphosphate; GGPS: Geranylgeranyl diphosphate synthase; GGS: L-glutamate gamma-semialdehyde; GO: Gene ontology; GPP: Geranyl diphosphate; GPS: Geranyl diphosphate synthase; GS: Glutamate synthase; GSEABase: Gene set enrichment analysis base; GST: Glutathione Stransferase; HB: Hunchback; HDIK: Ent-2-alpha-Hydroxyisokaurene; HDS: 4hydroxy-3-methylbut-2-enyl diphosphate synthase; HMBPP: 4-hydroxy-3methylbut-2-enyl diphosphate; HMG-CoA: Hydroxymethylglutaryl-Coenzyme A; HMGR: Hydroxymethylglutaryl- CoA reductase; HST: Shikimate Ohydroxycinnamoyltransferase; IAA: Indole-3-acetic acid; IDI: IPP isomerase IPP: Isopentenyl pyrophosphate; JA: Jasmonic acid; JAZ: Jasmonate ZIM domain-containing protein; KEGG: Kyoto encyclopedia of gene and genomes; KH: Ent-isokaurene C2-hydroxylase; KS: Kaurene synthase; LASPO: Iaspartate oxidase; MCT: 4-diphosphocytidyl-2-c-methyl-d-erythritol kinase; MEcPP: 2-C-methyl-D-erythritol 2,4-cyclodiphosphate; MEP: Methylerythritol phosphate; MEP: Methylerythritol phosphate; MJ: Methyl jasmonate; MVA: Mevalonicacid; NAC: An acronym for NAM, ATAF1-2, and CUC2; NCED: 9-cis-epoxycarotenoid dioxygenase; NON: 9'-cis-Neoxanthin; ODC: Ornithine decarboxylase; P5CS: Delta-1-pyrroline-5-carboxylate synthetase; PAL: Phenylalanine ammonia-lyase; PAO: Polyamine oxidase; PDT: Prephenate dehydratase; PFK: 6-phosphofructokinase 1; PFPA: Pyrophosphate-fructose-6-phosphate 1-phosphotransferase PInTFDB: Plant transcription factor database; PMD: Pimara-8(14), 15-diene; PMI: Mannose-6-phosphate isomerase; PP: Phosphatase; PRX: Peroxidase; PS: Phytoene synthase; PSY: Phytoene synthase; qPCR: Quantitative real-time PCR; RFS: Raffinose synthase; RNA-Seq: RNA-sequencing; RPKM: Reads per kilobase of exon model per million mapped reads; SA: Salicylic acid; SK: Shikimate kinase; STEM: Short time-series expression miner; SUS: Sucrose synthase; TFs: Transcription factors; TGA: TGACGTCA cis-element-binding protein; TPS: Trehalose 6-phosphate synthase; TREH: Alpha, alpha-trehalase; UHPLC-MS: Ultrahigh performance liquid chromatography-tandem mass spectroscopy; VON: 9-cis-Violaxanthin; ZEP: Zeaxanthin epoxidase

\section{Acknowledgments}

We thank Pengwei Hou and Dai Chen from Novel Bioinformatics Ltd., Co. for their technical assistance in bioinformatics analysis.

\section{Funding}

This work was supported by the National Natural Science Foundation of China (grant no. 31272041).

\section{Availability of data and materials}

The data sets supporting the results of this article are included within the article and its additional files.

\section{Authors' contributions}

$Y L, Q L$, and XW designed the study. QL and XW performed all the experiments. $Q L, X W, V T, J R, Y P$, and $Y L$ analyzed the data and wrote the manuscript. YP and YL provided experimental materials. All authors have read and approved the manuscript for publication.

\section{Competing interests}

The authors declare that they have no competing interests.

\section{Consent for publication}

Not applicable.

\section{Ethics approval and consent to participate}

Rice seeds used in this study were kindly provided by Prof. Yongjun Lin (Huazhong Agricultural University, Wuhan, China). Since the plant material was not collected from a wild source, no any permissions/permits were necessary. Larvae of C. suppressalis used in this study were retrieved from a laboratory colony that was maintained in our own laborartoy, and so far no any guildelines were adhered to for keeping the insects since they are common insect pests in rice fields.

\section{Author details}

${ }^{1}$ State Key Laboratory for Biology of Plant Diseases and Insect Pests, Institute of Plant Protection, Chinese Academy of Agricultural Sciences, Beijing, China. ${ }^{2}$ The French Associates Institute for Agriculture and Biotechnology of Drylands, The Jacob Blaustein Institute for Desert Research, Ben-Gurion University of the Negev, Sede Boger, Israel. ${ }^{3}$ Agroscope, Biosafety Research Group, Zurich, Switzerland.

Received: 26 July 2016 Accepted: 23 November 2016

Published online: 07 December 2016

\section{References}

1. Stam JM, Kroes A, Li Y, Gols R, van Loon JJ, Poelman EH, Dicke M. Plant interactions with multiple insect herbivores: from community to genes. Annu Rev Plant Biol. 2014:65(1):689-713.

2. Ray S, Gaffor I, Acevedo FE, Helms A, Chuang W, Tooker J, Felton GW, Luthe DS. Maize plants recognize herbivore-associated cues from caterpillar frass. J Chem Ecol. 2015;41(9):781-92.

3. Howe GA, Jander G. Plant immunity to insect herbivores. Annu Rev Plant Biol. 2008;59:41-66.

4. Dicke M, Baldwin IT. The evolutionary context for herbivore-induced plant volatiles: beyond the 'cry for help'. Trends Plant Sci. 2010;15(3):167-75.

5. Huang X, Chen J, Xiao H, Xiao Y, Wu J, Wu J, Zhou J, Zhang Y, Guo Y. Dynamic transcriptome analysis and volatile profiling of Gossypium hirsutum in response to the cotton bollworm Helicoverpa armigera. Sci Rep. 2015;5:11867.

6. Li J, Zhu L, Hull JJ, Liang S, Daniell H, Jin S, Zhang X. Transcriptome analysis reveals a comprehensive insect resistance response mechanism in cotton to infestation by the phloem feeding insect Bemisia tabaci (whitefly). Plant Biotechnol J. 2016. doi: 10.1111/pbi.12554

7. Tzin V, Fernandez-Pozo N, Richter A, Schmelz EA, Schoettner M, Schafer M, Ahern KR, Meihls LN, Kaur H, Huffaker A, et al. Dynamic maize responses to aphid feeding are revealed by a time series of transcriptomic and metabolomic assays. Plant Physiol. 2015;169(3):1727-43.

8. Zhou G, Wang X, Yan F, Wang X, Li R, Cheng J, Lou Y. Genome-wide transcriptional changes and defence-related chemical profiling of rice in response to infestation by the rice striped stem borer Chilo suppressalis. Physiol Plant. 2011;143(1):21-40.

9. Qi J, Sun G, Wang L, Zhao C, Hettenhausen C, Schuman MC, Baldwin IT, Li J, Song J, Liu Z, et al. Oral secretions from Mythimna separata insects specifically induce defense responses in maize as revealed by highdimensional biological data. Plant Cell Environ. 2016;39(8):1749-66.

10. Barah $\mathrm{P}$, Bones AM. Multidimensional approaches for studying plant defence against insects: from ecology to omics and synthetic biology. J Exp Bot. 2015;66(2):479-93

11. Zhou S, Lou YR, Tzin V, Jander G. Alteration of plant primary metabolism in response to insect herbivory. Plant Physiol. 2015;169(3):1488-98. 
12. Ehlting J, Chowrira SG, Mattheus N, Aeschliman DS, Arimura G, Bohlmann J. Comparative transcriptome analysis of Arabidopsis thaliana infested by diamond back moth (Plutella xylostella) larvae reveals signatures of stress response, secondary metabolism, and signalling. BMC Genomics. 2008;9:154.

13. Khush GS. What it will take to feed 5.0 billion rice consumers in 2030. Plant Mol Biol. 2005;59(1):1-6.

14. Lou Y, Zhang G, Zhang W, Hu Y, Zhang J. Biological control of rice insect pests in China. Biol Control. 2013;67(1):8-20.

15. Cohen MB, Chen M, Bentur JS, Heong KL, Ye G. Bt rice in Asia: potential benefits, impact, and sustainability. In: Romeis J, Shelton AM, Kennedy GG, editors. Integration of insect-resistant genetically modified crops within IPM programs. Dordrecht: Springer Science + Business Media B.V; 2008. p. 22348.

16. Liu Y, Wu H, Chen H, Liu Y, He J, Kang H, Sun Z, Pan G, Wang Q, Hu J, et al. A gene cluster encoding lectin receptor kinases confers broad-spectrum and durable insect resistance in rice. Nat Biotechnol. 2015;33(3):301-5.

17. Wang Y, Guo H, Li H, Zhang H, Miao X. Identification of transcription factors potential related to brown planthopper resistance in rice via microarray expression profiling. BMC Genomics. 2012;13:687.

18. Wang Y, Li H, Si Y, Zhang H, Guo H, Miao X. Microarray analysis of broadspectrum resistance derived from an indica cultivar Rathu Heenati. Planta. 2012;235(4):829-40.

19. Yang L, Zhang W. Genetic and biochemical mechanisms of rice resistance to planthopper. Plant Cell Rep. 2016;35(8):1559-72.

20. Hua H, Lu Q, Cai M, Xu C, Zhou D, Li X, Zhang Q. Analysis of rice genes induced by striped stemborer (Chilo suppressalis) attack identified a promoter fragment highly specifically responsive to insect feeding. Plant Mol Biol. 2007;65(4):519-30

21. Sun Y, Zhang Y, Cao G, Gu S, Wu K, Gao X, Guo Y. Rice gene expression profiles responding to larval feeding of the striped stem borer at the 1 st to 2nd instar stage. Insect Sci. 2011;18(3):273-81.

22. Han L, Li S, Liu P, Peng Y, Hou M. New artificial diet for continuous rearing of Chilo suppressalis (Lepidoptera: Crambidae). Ann Entomol Soc Am. 2012; 105(2):253-8.

23. Liu Z, Li X, Zhang J, Cai Y, Cheng T, Cheng C, Wang Y, Zhang C, Nie Y, Chen $Z$, et al. Autism-like behaviours and germline transmission in transgenic monkeys overexpressing MeCP2. Nature. 2016;530(7588):98-102.

24. Livak KJ, Schmittgen TD. Analysis of relative gene expression data using real-time quantitative PCR and the 2(-Delta Delta C(T)) method. Methods. 2001;25(4):402-8.

25. Jain M, Nijhawan A, Tyagi AK, Khurana JP. Validation of housekeeping genes as internal control for studying gene expression in rice by quantitative realtime PCR. Biochem Biophys Res Commun. 2006;345(2):646-51.

26. Kawahara Y, de la Bastide M, Hamilton J, Kanamori H, McCombie WR, Ouyang S, Schwartz D, Tanaka T, Wu J, Zhou S, et al. Improvement of the Oryza sativa Nipponbare reference genome using next generation sequence and optical map data. Rice. 2013;6(1):4

27. Wang K, Singh D, Zeng Z, Coleman SJ, Huang Y, Savich GL, He X, Mieczkowski P, Grimm SA, Perou CM, et al. MapSplice: accurate mapping of RNA-seq reads for splice junction discovery. Nucleic Acids Res. 2010;38(18):e178.

28. Wang L, Feng Z, Wang X, Wang X, Zhang X. DEGseq: an R package for identifying differentially expressed genes from RNA-seq data. Bioinformatics. 2010;26(1):136-8

29. Mortazavi A, Williams BA, McCue K, Schaeffer L, Wold B. Mapping and quantifying mammalian transcriptomes by RNA-Seq. Nat Meth. 2008;5(7):621-8

30. Benjamini $Y$, Hochberg $Y$. Controlling the false discovery rate: a practical and powerful approach to multiple testing. J R Stat Soc Series B Stat Methodol. 1995:57:289-300.

31. Usadel B, Nagel A, Steinhauser D, Gibon Y, Blasing OE, Redestig $H_{4}$ Sreenivasulu N, Krall L, Hannah MA, Poree F, et al. PageMan: an interactive ontology tool to generate, display, and annotate overview graphs for profiling experiments. BMC Bioinformatics. 2006;7:535.

32. Oliveros JC. VENNY. An interactive tool for comparing lists with Venn Diagrams. 2007.

33. Ernst J, Bar-Joseph Z. STEM: a tool for the analysis of short time series gene expression data. BMC Bioinformatics. 2006;7(1):191.

34. Volodarsky D, Leviatan N, Otcheretianski A, Fluhr R. HORMONOMETER: a tool for discerning transcript signatures of hormone action in the Arabidopsis transcriptome. Plant Physiol. 2009;150(4):1796-805.
35. Reimand J, Arak T, Vilo J. g:Profiler-a web server for functional interpretation of gene lists (2011 update). Nucleic Acids Res. 2011;39(Web Server issue): W307-15.

36. DeHaven CD, Evans AM, Dai H, Lawton KA. Organization of GC/MS and LC/ MS metabolomics data into chemical libraries. J Cheminform. 2010;2:9-9.

37. Kucharzewska P, Christianson HC, Belting M. Global profiling of metabolic adaptation to hypoxic stress in human glioblastoma cells. PloS one. 2015;10(1):e0116740

38. Pérez-Rodríguez P, Riaño-Pachón DM, Corrêa LGG, Rensing SA, Kersten B, Mueller-Roeber B. PInTFDB: updated content and new features of the plant transcription factor database. Nucleic Acids Res. 2010;38(Database issue):D822-7.

39. Tzin V, Galili G. New insights into the shikimate and aromatic amino acids biosynthesis pathways in plants. Mol Plant. 2010;3(6):956-72.

40. Appel HM, Fescemyer H, Ehlting J, Weston D, Rehrig E, Joshi T, Xu D, Bohlmann J, Schultz J. Transcriptional responses of Arabidopsis thaliana to chewing and sucking insect herbivores. Front Plant Sci. 2014;5:565.

41. Artico S, Ribeiro-Alves M, Oliveira-Neto OB, de Macedo LL, Silveira S, Grosside-Sa MF, Martinelli AP, Alves-Ferreira M. Transcriptome analysis of Gossypium hirsutum flower buds infested by cotton boll weevil (Anthonomus grandis) larvae. BMC Genomics. 2014;15:854.

42. Wang F, Ning D, Chen Y, Dang C, Han N, Liu Y, Ye G. Comparing gene expression profiles between $\mathrm{Bt}$ and non-Bt rice in response to brown planthopper infestation. Front Plant Sci. 2015;6:1181.

43. Wang $Y$, Wang $X$, Yuan $H$, Chen $R$, Zhu L, He R, He G. Responses of two contrasting genotypes of rice to brown planthopper. Mol Plant Microbe Interact. 2007;21(1):122-32

44. Dubey NK, Goel R, Ranjan A, Idris A, Singh SK, Bag SK, Chandrashekar K, Pandey KD, Singh PK, Sawant SV. Comparative transcriptome analysis of Gossypium hirsutum L. in response to sap sucking insects: aphid and whitefly. BMC Genomics. 2013;14:241

45. Giri MK, Swain S, Gautam JK, Singh S, Singh N, Bhattacharjee L, Nandi AK. The Arabidopsis thaliana At4g13040 gene, a unique member of the AP2/ EREBP family, is a positive regulator for salicylic acid accumulation and basal defense against bacterial pathogens. J Plant Physiol. 2014;171(10):860-7.

46. Hu L, Ye M, Li R, Zhang T, Zhou G, Wang Q, Lu J, Lou Y. The rice transcription factor WRKY53 suppresses herbivore-induced defenses by acting as a negative feedback modulator of mitogen-activated protein kinase activity. Plant Physiol. 2015;169(4):2907-21.

47. Li R, Zhang J, Li J, Zhou G, Wang Q, Bian W, Erb M, Lou Y. Prioritizing plant defence over growth through WRKY regulation facilitates infestation by non-target herbivores. eLife. 2015;4:e04805.

48. Wu J, Baldwin IT. New insights into plant responses to the attack from insect herbivores. Annu Rev Genet. 2010;44(1):1-24.

49. Lu J, Robert CA, Riemann M, Cosme M, Mene-Saffrane L, Massana J, Stout MJ, Lou Y, Gershenzon J, Erb M. Induced jasmonate signaling leads to contrasting effects on root damage and herbivore performance. Plant Physiol. 2015;167(3):1100-16.

50. Lou Y, Du M, Turlings TJ, Cheng J, Shan W. Exogenous application of jasmonic acid induces volatile emissions in rice and enhances parasitism of Nilaparvata lugens eggs by the parasitoid Anagrus nilaparvatae. J Chem Ecol. 2005;31(9):1985-2002

51. De Vleesschauwer D, Xu J, Höfte M. Making sense of hormone-mediated defense networking: from rice to Arabidopsis. Front Plant Sci. 2014;5:611.

52. De Vos M, Van Oosten VR, Van Poecke RM, Van Pelt JA, Pozo MJ, Mueller MJ, Buchala AJ, Metraux JP, Van Loon LC, Dicke M, et al. Signal signature and transcriptome changes of Arabidopsis during pathogen and insect attack. Mol Plant Microbe Interact. 2005;18(9):923-37.

53. Schwachtje J, Baldwin IT. Why does herbivore attack reconfigure primary metabolism? Plant Physiol. 2008;146(3):845-51.

54. Liu C, Hao F, Hu J, Zhang W, Wan L, Zhu L, Tang H, He G. Revealing different systems responses to brown planthopper infestation for pest susceptible and resistant rice plants with the combined metabonomic and gene-expression analysis. J Proteome Res. 2010;9(12):6774-85.

55. Scholz SS, Reichelt M, Mekonnen DW, Ludewig F, Mithofer A. Insect herbivory-elicited GABA accumulation in plants is a wound-induced, direct, systemic, and jasmonate-independent defense response. Front Plant Sci. 2015;6:1128.

56. Hagenbucher S, Olson DM, Ruberson JR, Wäckers FL, Romeis J. Resistance mechanisms against arthropod herbivores in cotton and their interactions with natural enemies. Crit Rev Plant Sci. 2013;32(6):458-82. 
57. Liu Q, Romeis J, Yu H, Zhang Y, Li Y, Peng Y. Bt rice does not disrupt the hostsearching behavior of the parasitoid Cotesia chilonis. Sci Rep. 2015;5:15295.

58. Tholl D. Biosynthesis and biological functions of terpenoids in plants. Adv Biochem Eng Biotechnol. 2015;148:63-106.

59. Matsye PD, Kumar R, Hosseini P, Jones CM, Tremblay A, Alkharouf NW, Matthews BF, Klink VP. Mapping cell fate decisions that occur during soybean defense responses. Plant Mol Biol. 2011;77:513-28.

60. Matsye PD, Lawrence GW, Youssef RM, Kim K-H, Matthews BF, Lawrence KS, Klink VP. The expression of a naturally occurring, truncated allele of an aSNAP gene suppresses plant parasitic nematode infection. Plant Mol Biol. 2012:80:131-55.

61. Matthews BF, Beard H, MacDonald MH, Kabir S, Youssef RM, Hosseini P, Brewer E. Engineered resistance and hypersusceptibility through functional metabolic studies of 100 genes in soybean to its major pathogen, the soybean cyst nematode. Planta. 2013;237:1337-57.

Submit your next manuscript to BioMed Central and we will help you at every step:

- We accept pre-submission inquiries

- Our selector tool helps you to find the most relevant journal

- We provide round the clock customer support

- Convenient online submission

- Thorough peer review

- Inclusion in PubMed and all major indexing services

- Maximum visibility for your research

Submit your manuscript at www.biomedcentral.com/submit
Biomed Central 